\title{
Differences in expression of virulence genes amongst invasive and colonizing isolates of meticillin-resistant Staphylococcus aureus
}

The prevalence of meticillin-resistant Staphylococcus aureus (MRSA) is an important global concern in healthcare due to potentially life-threatening infections and difficulties in treatment. The organism can colonize the mucosa, e.g. anterior nares, or cause invasive infections, e.g. bloodstream infections, through the coordinated expression of extracellular and cell-bound virulence factors (Goerke et al., 2000; Novick et al., 1993). The transcription profiles of virulence genes vary in vivo depending on the dynamic interaction between the host environment and the pathogen. Since the dynamic environment may be differentially altered in MRSA carriage compared to infection, the expression of $S$. aureus virulence genes may also vary. We previously found no significant correlation between the presence of 17 virulence genes and invasiveness of MRSA isolates (O’Donnell et al., 2008) and this nonassociation of specific genes or combinations of genes with invasive isolates is in agreement with other larger studies (Lindsay et al., 2006). The lack of a definitive correlation between invasive $S$. aureus strains and the carriage of virulence genes suggests that the expression, rather than carriage of virulence determinants in vivo, may mediate pathogenicity.

We investigated the in vitro expression of selected virulence genes, gammahaemolysin $(h l g)$, collagen adhesionencoding gene (cna) and staphylococcal enterotoxin A (sea), in colonizing compared with invasive MRSA clinical isolates, using the same isolate collection used in our previous study (O'Donnell et al., 2008). hlg and sea were representative of toxin genes and cna was representative of adhesion genes. These genes were selected because they were among those present in all isolates from our previous study. We also investigated the expression of one regulatory gene, RNAIII, the effector molecule of the accessory gene regulator (agr) system which is reported to upregulate toxin genes and downregulate adhesion genes.

Twenty isolates were recovered from patients with device-related bloodstream infections between January 2002 and June 2005 and 20 isolates were from nasal swabs collected from patients that were MRSApositive on first hospital admission (between September 2006 and June 2007) but with no apparent signs of infection. The spa type of each isolate was used to infer the sequence type (ST) or clonal complex from which the isolate originated and the staphylococcal cassette chromosome mec type was also determined (O’Neill et al., 2007). RNA was isolated from cultures grown to an optical density of 2 (3-5 h, determined from preliminary experiments to correspond to the mid-late exponential phase) in brain heart infusion broth supplemented with commercially available $5 \%$ pooled human serum to mimic the in vivo environment. Real-time RT-PCR was performed using specific primers for each of the four genes and expression levels were normalized to the expression of $g y r B$ as an internal control. The absolute quantity of each gene in isolates could not be determined without appropriate standards, therefore calibrator-normalized relative quantification was used. The relative expression level of each gene was the ratio of its normalized value in the test isolates to its normalized value in isolate $\mathrm{BH} 16$. Isolate $\mathrm{BH} 16$ was chosen arbitrarily from the collection as the calibrator isolate.

The relative expression levels of the four virulence genes in invasive versus colonizing isolates (including the gene regulator RNAIII) are shown in Fig. 1. There was a high level of variation in the relative expression of virulence genes among both groups of isolates. Comparing the two groups, colonizing and invasive, there was no significant alteration in the transcription of RNAIII in invasive isolates versus colonizing isolates $(P=0.0859$ by
Mann-Whitney test using GraphPad Prism version 4.00 for Windows). Transcription of cna was upregulated in colonizing isolates compared to invasive isolates $(P=0.0110)$ whereas transcription of $h l g$ was upregulated in invasive isolates compared to colonizing isolates $(P=0.0193$ by Mann-Whitney). Although more invasive isolates than colonizing isolates expressed relatively higher levels of the sea transcript, this was not statistically significant $(P=0.1456)$. Comparing the relative expression of all four genes in each isolate (data not shown), the transcription of RNAIII was accompanied by a reduction in transcription of cna and increased transcription of $h l g$ in only four invasive isolates and none of the colonizing isolates. RNAIII did not increase transcription of sea but the sea transcript was apparently expressed at low levels in most isolates or was not detectable. Genotyping of isolates used in this study revealed that both colonizing and invasive groups were highly clonal, belonging to either ST22-MRSA-IV $(100 \%$ and $65 \%)$ or ST8-MRSA-II (0\% and $35 \%$ ). Of the four agr types that have been described, ST22-MRSA-IV and ST8MRSA-II isolates belong to agr type 1 (Monecke et al., 2008). Fig. 2 shows that within the invasive isolates group, ST8MRSA-II isolates differed significantly in their transcription levels to ST22-MRSAIV for cna $(P=0.005)$ and sea $(P=0.019)$ but not for $h \lg (P=0.064)$ or RNAIII $(P=0.062)$. When the ST8-MRSA-II isolates were omitted from the overall analysis, only the transcript levels of sea were significantly increased $(P=0.013)$ in the invasive group compared to the colonizing isolates. Although in the invasive isolates the transcript levels of $c n a$ were decreased and those of $h l g$ were increased, these failed to reach significance $(P=0.22$ and $P=0.19)$ when only ST22-

MRSA-IV isolates were analysed (data not shown).

Virulence factors that promote binding to the mucosal layers are important in $S$. 


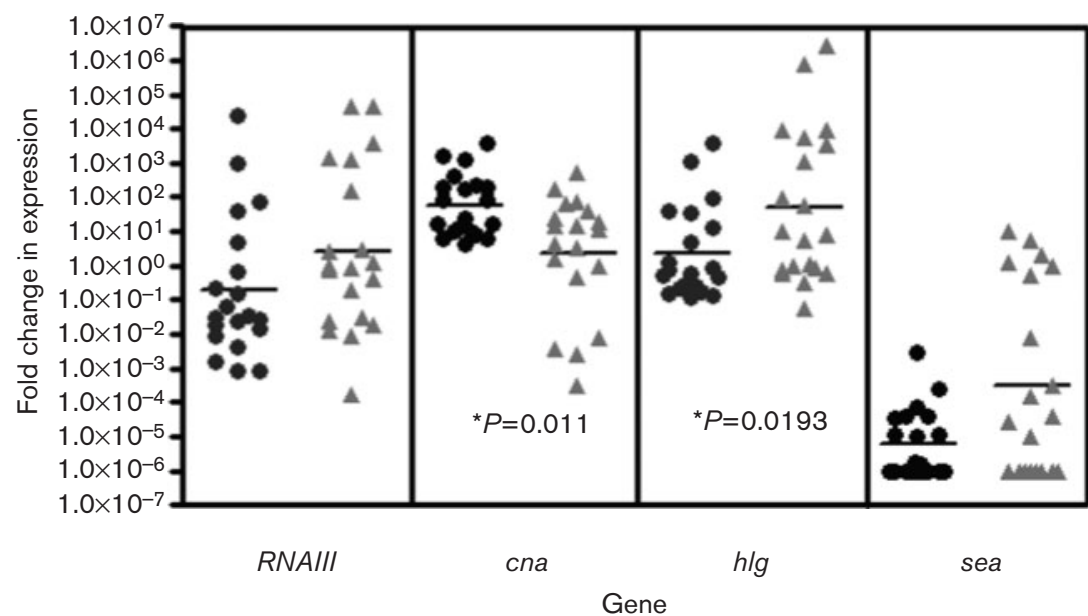

Fig. 1. Fold change in transcript levels of RNAIII, cna, hlg and sea in MRSA colonizing isolates (black circles) and invasive isolates (grey triangles) using real-time PCR with relative quantification with respect to an arbitrarily chosen isolate. Horizontal lines indicate the median value for each dataset. Statistically significant changes in transcript levels are indicated. aureus colonization of the host, whereas factors that promote tissue damage and provoke damaging immune responses are important during $S$. aureus infection (Cheung et al., 2004; Novick et al., 1993). In the present study, we found significant differences in the relative expression levels of virulence genes in colonizing versus invasive MRSA isolates when the clinical isolate was grown to mid-late exponential phase in the presence of human serum in an attempt to mimic the in vivo conditions. In the presence of human serum, a greater number of invasive isolates expressed lower levels of the cellassociated transcript cna and higher levels

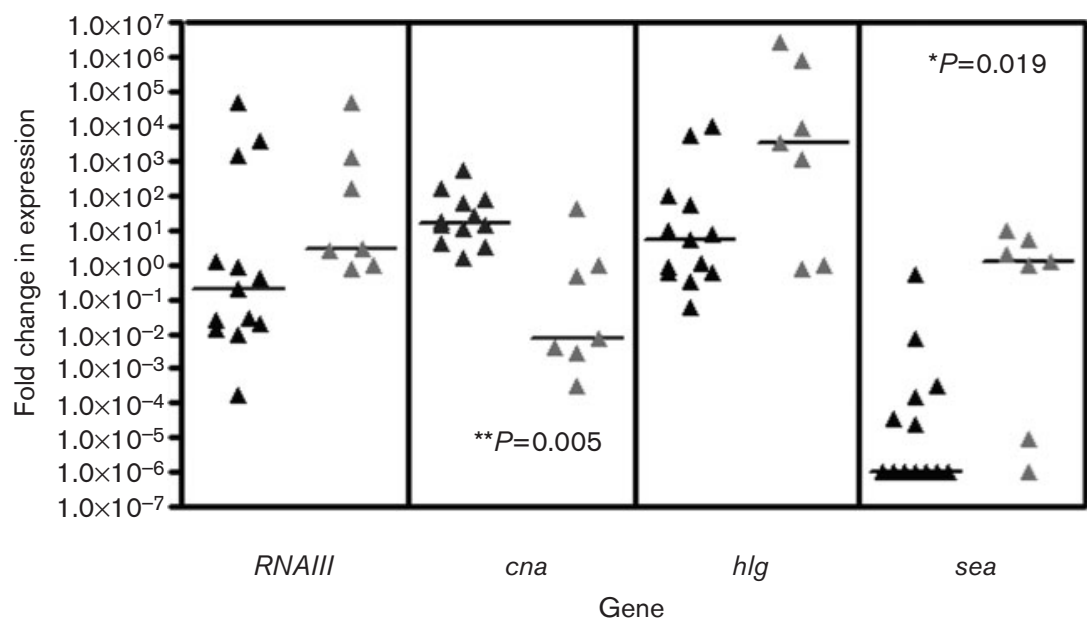

Fig. 2. Fold changes in transcript levels of RNAIII, cna, hlg and sea in MRSA invasive isolates when correlated with the clonal type of the isolate, ST22 (black triangles) and ST8 (grey triangles). Horizontal lines indicate the median value for each dataset. Statistically significant changes in transcript levels are indicated. of the toxin gene hlg but not of sea. A greater number of colonizing isolates expressed relatively high transcript levels of cna with hlg transcripts also being relatively high in some, and sea transcription was low or beyond the limit of detection. Our data also indicate that the origin of the isolate cannot be considered independently of other genetic factors relating to the isolate as these may make a significant contribution to pathogenicity. It has been shown that the carriage of virulence genes is highly clonal but here we have also demonstrated an association between clonal type and transcription of virulence genes (Fig. 2). Although the differences in the pattern of expression of virulence-associated genes may contribute to the pathogenicity of MRSA in invasive versus colonizing isolates, this study shows that colonizing isolates may also have the capacity to promote host damage in some situations. Alterations in the local environment, such as $\mathrm{pH}$ or proteolytic activity, may affect the activity of expressed proteins, or differences in the immune response to the organism may determine the overall pathogenesis of MRSA.

Expression of RNAIII, in the lateexponential phase, positively correlated with the expression of $h l g$ and negatively correlated with the expression of cna for only a small number of invasive isolates. Similarly, other authors have shown that positive correlation between transcription of the toxin genes and the regulator gene RNAIII may be isolate-specific (Papakyriacou et al., 2000; Sabersheikh \& Saunders, 2004) or may vary with the phase of growth studied as RNAIII is a quorum-sensing effector. Alternatively, expression of RNAIII may not be critical for expression of $S$. aureus toxins and this has been confirmed in one of the few studies that have measured in vivo transcription of RNAIII in an animal model of infection (Yarwood et al., 2002). It is also possible that virulence gene expression may be regulated by other regulator genes or a combination of these. Alternative regulatory systems identified in S. aureus include sar, sigB, rot, arlR/S, svrA and saeR/S (Bronner et al., 2004; Novick \& Jiang, 2003).

We have found that under in vitro conditions using human serum that may 
partly mimic what occurs in patients (at least in infection), variations in the transcription of some virulence genes occurred that correlated with the isolate source (colonizing or from infection), and within the invasive group, ST8-MRSA-II isolates showed decreased transcription of cna and increased transcription of sea compared to ST22-MRSA-IV isolates. Because environmental conditions in the nares of patients differ from those in the bloodstream, further work in this area should aim to mimic more closely the local host environment during colonization and infection (e.g. bacterial growth phase, $\mathrm{pH}$, osmolarity and/or host-niche-specific factors, e.g. presence of polymorphonuclear neutrophils and/or platelets) or to investigate transcription of virulence genes when isolates are grown in the presence of the patient's own serum. These studies should also be extended to other virulence genes and other gene regulators. Accurate direct measurement of gene expression levels in vivo presents a challenge, but this research area may benefit from recent improvements in microgenomic detection of bacterial RNA in human tissues (Klitgaard et al., 2007). These approaches should further our understanding of the properties of $S$. aureus that allow it to transform from transient commensal to pathogen.

\section{Acknowledgements}

This work was funded partly by a Summer Research Grant from the Royal College of Surgeons in Ireland and was also supported by the Association of Physicians of Great Britain and Ireland, the Charitable Infirmary
Charitable Trust and the Madeleine Farrell Charitable Bequest.

\section{Amalina Abu Othman, ${ }^{1}$ Hilary Humphreys, ${ }^{1,2}$ Eoghan O'Neill ${ }^{1,3}$ and Deirdre Fitzgerald-Hughes ${ }^{1}$}

${ }^{1}$ Department of Clinical Microbiology, Royal College of Surgeons in Ireland, Smurfit Building, Beaumont Hospital, Dublin 9, Ireland

${ }^{2}$ Department of Microbiology, Beaumont Hospital, Dublin 9, Ireland

${ }^{3}$ Department of Microbiology, Connolly Hospital, Dublin 15, Ireland

\section{Correspondence:}

Deirdre Fitzgerald-Hughes

(dfitzgeraldhughes@rcsi.ie)

Bronner, S., Monteil, H. \& Prevost, G. (2004). Regulation of virulence determinants in Staphylococcus aureus: complexity and applications. FEMS Microbiol Rev 28, 183-200.

Cheung, A. L., Bayer, A. S., Zhang, G., Gresham, H. \& Xiong, Y. Q. (2004). Regulation of virulence determinants in vitro and in vivo in Staphylococcus aureus. FEMS Immunol Med Microbiol 40, 1-9.

Goerke, C., Campana, S., Bayer, M. G., Doring, G., Botzenhart, K. \& Wolz, C. (2000). Direct

quantitative transcript analysis of the agr regulon of Staphylococcus aureus during human infection in comparison to the expression profile in vitro. Infect Immun 68, 1304-1311.

Klitgaard, K., Jensen, T. K., Angen, O. \& Boye, M. (2007). Measurement of bacterial gene expression in vivo by laser capture microdissection and quantitative real-time RTPCR. J Microbiol Methods 69, 414-416.

Lindsay, J. A., Moore, C. E., Day, N. P., Peacock, S. J., Witney, A. A., Stabler, R. A., Husain, S. E., Butcher, P. D. \& Hinds, J. (2006). Microarrays reveal that each of the ten dominant lineages of
Staphylococcus aureus has a unique combination of surface-associated and regulatory genes. J Bacteriol 188, 669-676.

Monecke, S., Slickers, P. \& Ehricht, R. (2008). Assignment of Staphylococcus aureus isolates to clonal complexes based on microarray analysis and pattern recognition. FEMS Immunol Med Microbiol 53, 237-251.

Novick, R. P. \& Jiang, D. (2003). The staphylococcal saeRS system coordinates environmental signals with agr quorum sensing. Microbiology 149, 2709-2717.

Novick, R. P., Ross, H. F., Projan, S. J., Kornblum, J., Kreiswirth, B. \& Moghazeh, S. (1993). Synthesis of staphylococcal virulence factors is controlled by a regulatory RNA molecule. EMBO J 12, 3967-3975.

O'Donnell, S., Humphreys, H. \& Hughes, D. (2008). Distribution of virulence genes among colonising and invasive isolates of meticillinresistant Staphylococcus aureus. Clin Microbiol Infect 14, 625-626.

O'Neill, E., Pozzi, C., Houston, P., Smyth, D., Humphreys, H., Robinson, D. A. \& O'Gara, J. P. (2007). Association between meticillin susceptibility and biofilm regulation in Staphylococcus aureus isolates from devicerelated infections. J Clin Microbiol 45, 1379 1388.

Papakyriacou, H., Vaz, D., Simor, A., Louie, M. \& McGavin, M. J. (2000). Molecular analysis of the accessory gene regulator (agr) locus and balance of virulence factor expression in epidemic meticillin-resistant Staphylococcus aureus. $J$ Infect Dis 181, 990-1000.

Sabersheikh, S. \& Saunders, N. A. (2004). Quantification of virulence-associated gene transcripts in epidemic meticillin resistant Staphylococcus aureus by real-time PCR. Mol Cell Probes 18, 23-31.

Yarwood, J. M., McCormick, J. K., Paustian, M. L., Kapur, V. \& Schlievert, P. M. (2002). Repression of the Staphylococcus aureus accessory gene regulator in serum and in vivo. J Bacteriol 184, 1095-1101. 